\begin{tabular}{|c|c|}
\hline Title & Comprehensive Synthesis of Photoreactive Phenylthiourea Derivatives for the Photoaffinity L abeling \\
\hline Author(s) & $\begin{array}{l}\text { Ishida, A kiko; W ang, Lei; T achrim, Zetryana Puteri; Suzuki, T akeyuki; Sakihama, Y asuko; Hashidoko, Y asuyuki; } \\
\text { Hashimoto, Makoto }\end{array}$ \\
\hline Citation & $\begin{array}{l}\text { Chemistry Select, 2(1), 160-164 } \\
\text { https://doi.org/10.1002/slct.201601675 }\end{array}$ \\
\hline Issue Date & 2017-01-10 \\
\hline Doc URL & http://hdl.handle.net/2115/68065 \\
\hline Rights & $\begin{array}{l}\text { This is the peer reviewed version of the following article:"Comprehensive Synthesis of Photoreactive Phenylthiourea } \\
\text { Derivatives for the Photoaffinity L abeling", Chemistry Select 2(1), pp160-164, 2017, which has been published in final } \\
\text { form at http://dx.doi.org/10.1002/slct.201601675. This article may be used for non-commercial purposes in accordance } \\
\text { With Wiley-V CH Terms and Conditions for self-archiving }\end{array}$ \\
\hline Tyре & article (author version) \\
\hline File Information & 3 ChemistrySelect 2 160-164.pdf \\
\hline
\end{tabular}

Instructions for use 


\title{
Comprehensive Synthesis of Photoreactive Phenylthiourea Derivatives for the Photoaffinity Labeling
}

\author{
Akiko Ishida, ${ }^{[a]}$ Lei Wang ${ }^{[a]}$ Zetryana Puteri Tachrim, ${ }^{[a]}$ Takeyuki Suzuki,${ }^{[b]}$ Yasuko Sakihama, ${ }^{[a]}$ Yasuyuki \\ Hashidoko, ${ }^{[\mathrm{a}]}$ and Makoto Hashimoto*[a]
}

\begin{abstract}
Phenylthiourea (PTU) is well known as bioactive compound and one of the reversible inhibitors for tyrosinase. Tyrosinase plays very important roles for tyrosine metabolisms to maintain the skin homeostasis from sunlight with forming melanin. Synthesis of photoaffinity label reagents of PTU will be attractive thesis to elucidate functional analysis for tyrosinase.
\end{abstract}

\section{Introduction}

Phenylthiourea (PTU) and its synthetic derivatives are wellknown as bioactive compounds that have high affinity for urea channels in human red cells, ${ }^{[1]}$ bitter tastants ${ }^{[2]}$ and tyrosinase inhibitors. ${ }^{[3]}$ Tyrosinase is an enzyme that oxidizes the phenolic part of tyrosine, forming dihydroxyphenylalanine (DOPA) from tyrosine and further oxidizing of DOPA to dopaquinone. Dopaquinone plays an important role in melanin formation in sunburns. Inhibition of melanin formation thus finds promising applications in skin-whitening (i.e., sun protection). ${ }^{[4]}$ PTU derivatives are well-known reversible inhibitors for tyrosinase. The effects of substitution on aromatic rings inhibit tyrosinase have been reported. $^{[3]}$ Photoaffinity labeling ${ }^{[5]}$ is a useful biochemical method for analysis biological interactions between low-molecular-weight bioactive compounds and biomolecules. The methodology may afford other information near the binding sites, which cannot obtain from structure-activity relationship studies. A few studies have elucidated biological activities on the synthesis of azide ${ }^{[6 ., 7]}$ and benzoyl (benzophenone) ${ }^{[8]}$ attached to the aromatic ring of PTU. However no study on typical photophores with their biological activities has been reported. In this paper, we describe the comprehensive synthesis of photoreactive PTU derivatives (Fig.1), which we then used in an inhibitory activity assay for tyrosinase.

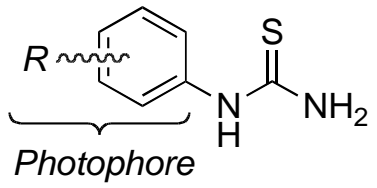

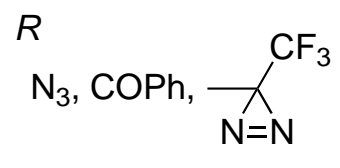

Figure 1. Schematic representation for photoreactive phenylthiourea (PTU) derivatives

[a] A. Ishida, Dr. L. Wang, Z. P. Tachrim, Dr. Y. Sakihama, Prof. Dr. Y Hashidoko, Dr. M. Hashimoto, Division of Applied Science, Graduate School of Agriculture, Hokkaido University; Kita 9, Nishi 9, Kita-ku, Sapporo 060-8589, Japan, E-mail: hasimoto@abs.agr.hokudai.ac.jp. Homepage: http://www.agr.hokudai.ac.jp/en/

[b] Dr. T. Suzuki, Division of Applied Science, The Institute of Scientific and Industrial Research, Osaka University, Mihogaoka, Ibaraki-shi, Osaka 567-0047, Japan

Supporting information for this article is given via a link at the end of the document.

\section{Results and Discussion}

Azide PTU derivatives: Lamotte et al. reported the synthesis of 4-azide PTU from the corresponding 4-nitro PTU derivative, but they did not give a detailed description of the synthesis. ${ }^{[6]}$ Another study constructed the 3-azide-4-chloro PTU derivative by using ammonia and an isothiocyanate moiety. This route utilized thiophosgene to produce isothiocyanate. ${ }^{[7]}$ Our synthetic strategies for both 3- and 4- isomers are identical and do not use highly toxic reagents. We reduced 3- (1a) or 4- (1b) nitro substituted PTU with iron under acidic conditions to form aniline derivatives ( $\mathbf{2} \mathbf{a}$ and $\mathbf{b})$. The aniline derivatives were subjected to diazotization followed by azidation to form phenylazide derivatives (3a and $\mathbf{b}$ ) at moderate yields (Scheme 1).

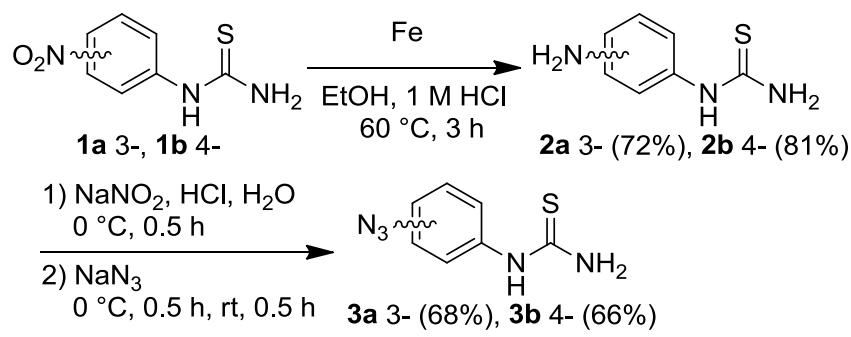

Scheme 1. Synthesis of phenylazide derivatives of phenylthioureas (3a and 3b)

Benzophenone PTU derivatives: Kamel et al. reported that 4aminobenzophenone reacts with potassium thiocyanate,forming a PTU derivative. ${ }^{[8]}$ In our preliminary experiments, however, $m$ toluidine did not react under the conditions they used. To establish general methods for the preparation of 3- (4a) or 4(4b) aminobenzophenones were converted to benzoyl-protected thiourea derivatives by using ammonium thiocyanate and benzoyl chloride ${ }^{[9]}$, and then deprotected in alkali to afford benzophenone derivatives of PTU (5a and $\mathbf{5 b})$ at moderate yields (Scheme 2).

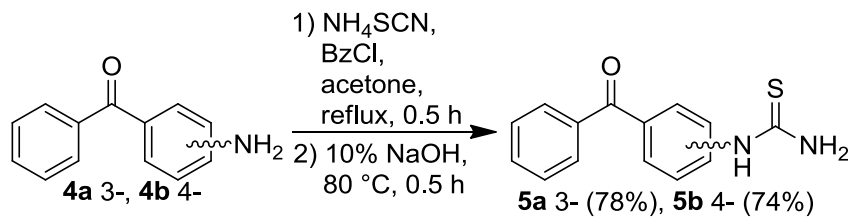

Scheme 2. Synthesis of benzophenone derivatives of phenylthioureas (5a and 5b)

3-trifluoromethyldiazirinyl (3-TFMD) derivatives: A few papers report the synthesis of 3-TFMD-aniline ${ }^{[10-12]}$, which is a precursor for PTU derivatives. A common method using formamide-protected trifluoroacetophenone has been utilized to protect the aniline group. ${ }^{[10]}$ After construction of TFMD, the formyl group was deprotected under acidic conditions, and the 


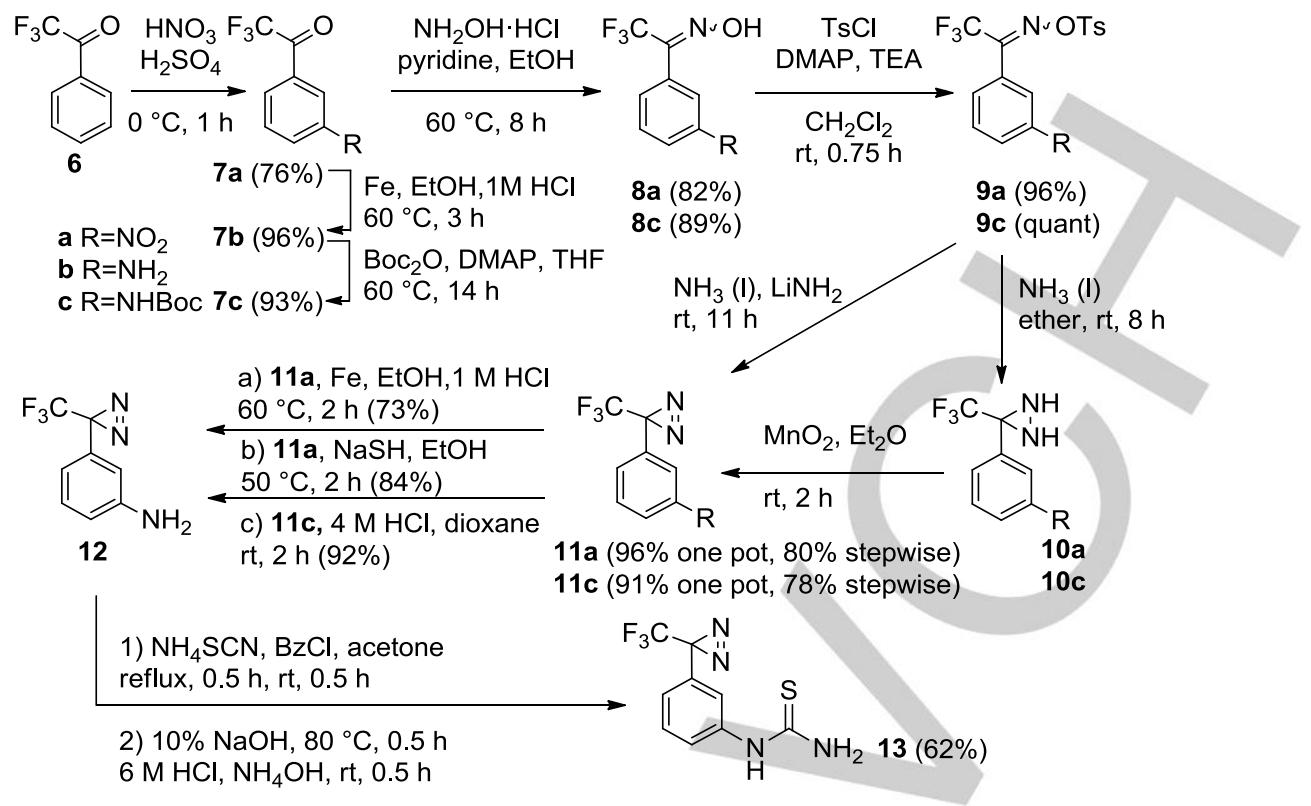

Scheme 3. Synthesis of 3-trifluoromethyldiazirinyl derivative of phenylthiourea (13) from 2,2,2-trifluoroacetophenone derivatives 7a and 7c

amine generated in situ was utilized for next reaction without further purifications. ${ }^{[11]}$ Previous yields of diazirine from synthesis using four steps are very low (less than $30 \%$ each steps, over all yield was $0.6 \%)^{[10]}$, and the efficiency of deprotection of the formamide moiety is not established (less than $70 \%$ ). ${ }^{[11 \mathrm{c}]}$

Another method for generating the aniline skeleton is the reduction of nitorobenzene derivatives. The previously reported procedures contained several obscure points. (For example, the yields is over $>100 \%$ chemical yields for 2 steps), and that of the a reduction of 3-nitrophenyl-TFMD to 3-aminophenyl-TFMD was is less than $50 \%{ }^{[12]}$

On the basis of the reported procedures, we studied the synthesis of 3-aminophenyl-TFMD and attempted it to prepare PTU derivatives. We selected the nitro and NHBoc groups to compare the synthesis of 3-aminophenyl-TFMD. Trifluoroacetophenone $\mathbf{6}$ was nitrated to produce 3-nitro trifluoroacetophenone $\mathbf{7 a}$ and the minor product, its 2-nitro isomer $(<15 \%)$. Reduction of $\mathbf{7 a}$ using iron followed by Boc protection led to the formation of 3-Boc-aminophenyl derivatives 7c. Compounds $7 \mathbf{a}$ and $7 \mathrm{c}$ were converted were converted to oximes ( $8 \mathbf{a}$ and $\mathbf{c}$ ) and $O$-tosyloxime derivatives (9a and $\mathbf{c})$. The $O$-Tosyloximes were then subjected to stepwise conversion ${ }^{[13]}$ to 10 and 11, and 9 was directly converted 11 via one-pot synthesis ${ }^{[14]}$ to construct the TFMD moiety. One-pot conversions led to yields higher than those of the stepwise methods. Reductions of nitro group with iron or sodium bisulfide led to the formation of 3-nitrophenyl-TFMD (11a) and 3-aminophenylTFMD (12) with satisfactory yields. Treatment of Bocaminophenyl-TFMD (11c) under acidic conditions produced 12, which was stable at $-20^{\circ} \mathrm{C}$ for at least 1 month, at $>90 \%$ yield. Our improvements thus promoted the synthesis of 3aminophenyl-TFMD 12 at $>60 \%$ yield from acetophenone derivatives ( $7 \mathbf{a}$ and $7 \mathrm{c})$.

Compound 12 was treated with ammonium thiocyanate and benzoyl chloride under reflux conditions to form benzoyl PTU derivatives. Alkaline hydrolysis of the reaction mixture produced
3-TFMD-modified PTU 13, which retains the TFMD moiety, at moderate yield (Scheme 3).

4-Trifluoromethyldiazirinyl (4-TFMD) derivatives: A few studies have performed the synthesis of 4-aminophenyl substituted TFMD because of its instability arising from the structure of the nitrogen lone pair, which can be easily conjugated with TFMD via the benzene ring. We therefore used the precursors nitro (16a), fluoride (16b), azide (16c), phthalimide (16d) and tert-butyloxycarbonyl protected amine (16e) to synthesis 4-aminophenyl-TFMD.

4-Nitrobenzaldehyde 14 was treated with (trifluoromethyl)trimethylsilane, and then subjected to DessMartin oxidation to form the trifluoroacetophenone derivative 16a. Synthesis of the azide (16b), phthalimide (16c) and NHBoc (16e)-substituted trifluoroacetophenone derivatives from 4fluoro-trifluoroacetophenone (16b) had moderate yields. These trifluoroacetophenone derivatives were then used in a subsequent reaction. General methods for the synthesis of diazirine using 16a-c and e were performed. ${ }^{[13]}$ Partial deprotection of phthalimide group of $\mathbf{1 6 d}$ formed the corresponding amine, occurring alongside both oximiation and diaziridine formation The isolated phathalimide derivatives $\mathbf{1 7 d}$ had lower yield (36\%), but one-pot three-step reaction of trifluoroacetyl derivative $\mathbf{1 6 d}$ to 4-aminophenyltrifluoromethyldiaziridine (19d) resulted in $61 \%$ yield. Compound 19d was easily oxidized to diaziridine 21 at $>85 \%$ yield. Other substituted diaziridines (19a c and e) were oxidized to the corresponding diazirines (20a c and e) with good yields, and then converted to amines.

Amine conversion of the fluoride derivative $20 \mathrm{~b}$ in $\mathrm{NH}_{4} \mathrm{OH}$ at $100{ }^{\circ} \mathrm{C}$ resulted in a complex mixture. The 4-fluorophenyldiaziridine (19b) also formed a complex mixture under the same conditions. Reduction of the 4-nitro derivatives (20a) with iron and reduction of the 4-azide (20c) TFMD derivatives using 


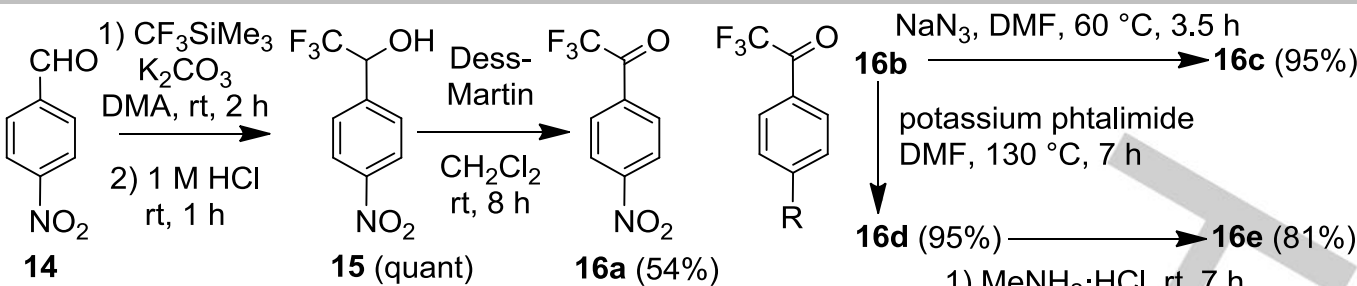

1) $\mathrm{MeNH}_{2} \cdot \mathrm{HCl}, \mathrm{rt}, 7 \mathrm{~h}$

2) $\mathrm{Boc}_{2} \mathrm{O}, \mathrm{DMAP}, 60^{\circ} \mathrm{C}, 14 \mathrm{~h}$

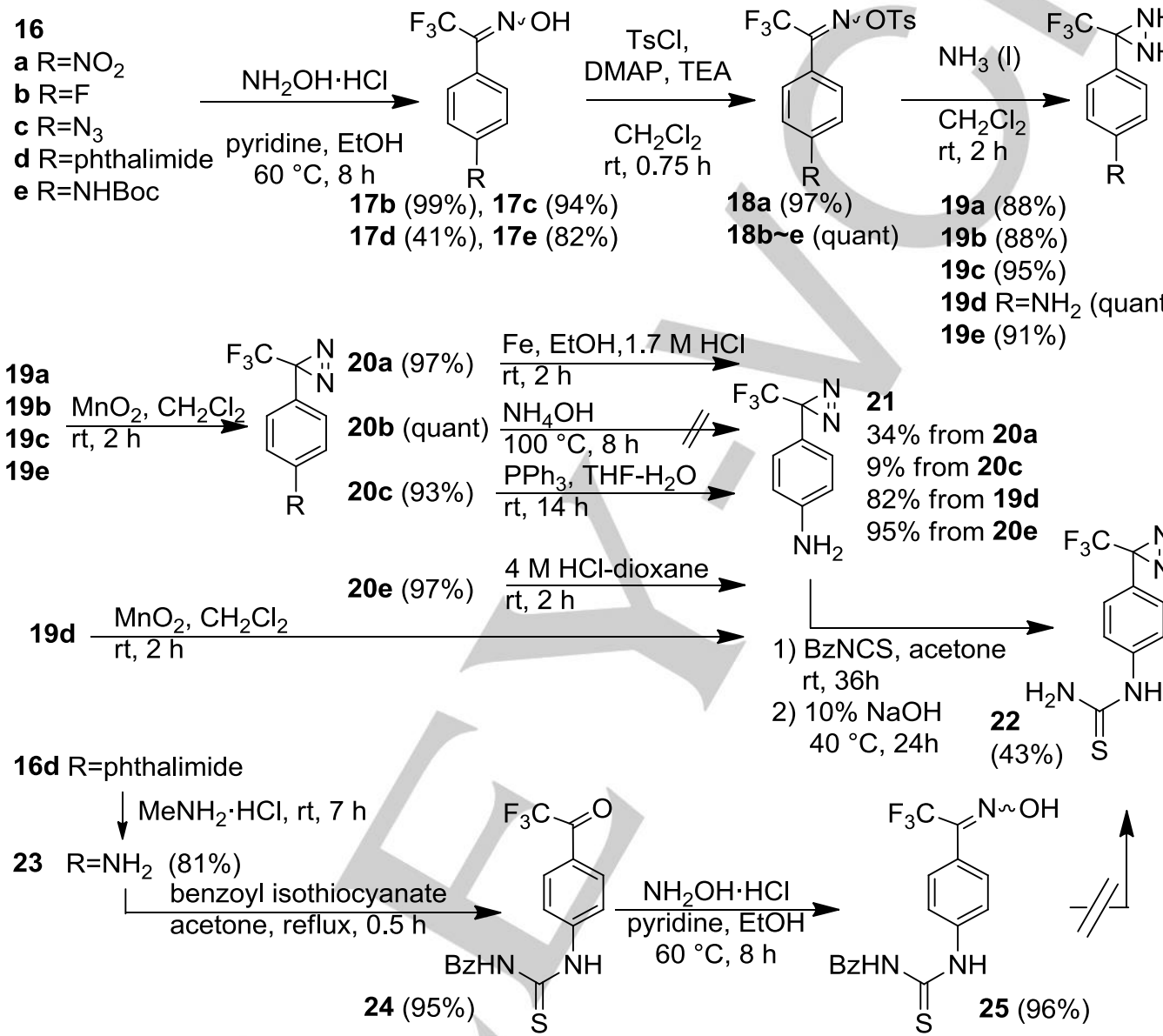

Scheme 4. Synthesis of 4-trifluoromethyldiazirinyl derivative of phenylthiourea (22) from 2,2,2-trifluoroacetophenone derivatives 16a 16e

triphenylphosphine did not result in satisfactory yields because of the broken TFMD moiety during the reaction. Deprotection of the Boc group of $20 \mathrm{e}$ resulted in 4 -aminophenyl-TFMD $(\mathbf{2 1}$; > $90 \%$ yield). Very recently, similar studies have been done to synthesize $21,{ }^{[15]}$ but the total yields of $\mathbf{2 1}$ from the common intermediates $16 \mathrm{~b}$ via $16 \mathrm{e}(>40 \%)$ are more than twice those reported in these studies.

Compound 21 was used in thiourea formation. Refluxing with $\mathrm{NH}_{4} \mathrm{SCN}$ and benzoyl chloride, which is identical to that used in the synthesis of 5 and 13, promoted decomposition of TFMD, converting the aniline moiety was to urea during the reaction. Benzoyl isothiocyanate was used in the reaction to enable it to proceed at a lower temperature. The reaction at room temperature produced 4-TFMD-PTU 22 in moderate yield without decomposition of the TFMD moiety.
We also attempted to construct TFMD using PTU derivatives. 4Trifluoroacetylaniline (23), which was easily prepared from phthalimide derivatives $\mathbf{1 6 d}$, was converted to benzoylthiourea derivatives 24. Although the oximation of $\mathbf{2 4}$ occurred smoothly, tosylation of oxime $\mathbf{2 5}$ could not proceed in the general methods. Mesitylation, which has reactivity greater than that of tosylation, also did not be afforded desired products.

Photoreactivity of synthesized PTU derivatives: The synthesized PTU azides ( $\mathbf{3} \mathbf{a}$ and $\mathbf{3} \mathbf{b}$ ) derivatives were to photoirradiate in methanol using a mercury lamp. Similarly, the derivatives benzophenones ( $\mathbf{5 a}$ and $\mathbf{5 b}$ ) and diazirines ( $\mathbf{1 3}$ and 22) were exposed using a black light. Each photolyzed mixture was analyzed by mass spectrometry. Methanol adduct signals for all samples were measured after 1 hour irradiation, and UV spectra for the diazirines were subjected to time-course analysis 


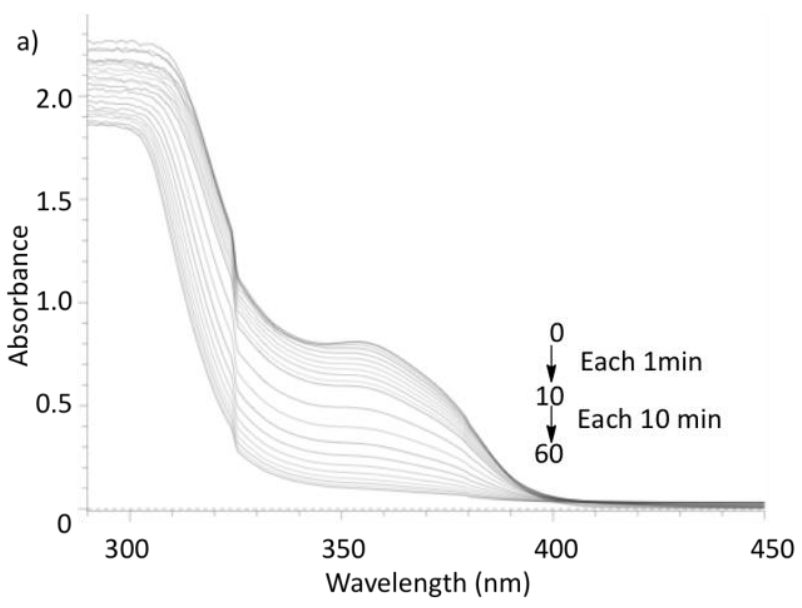

b)

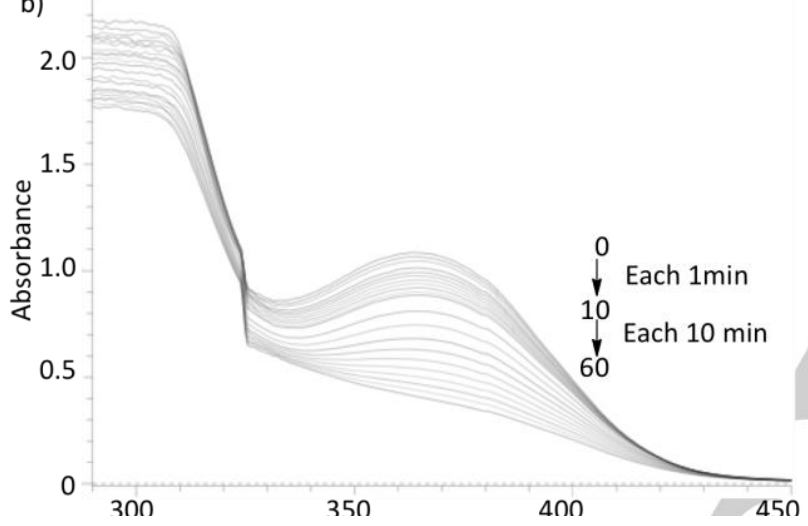

A) Tyrosine

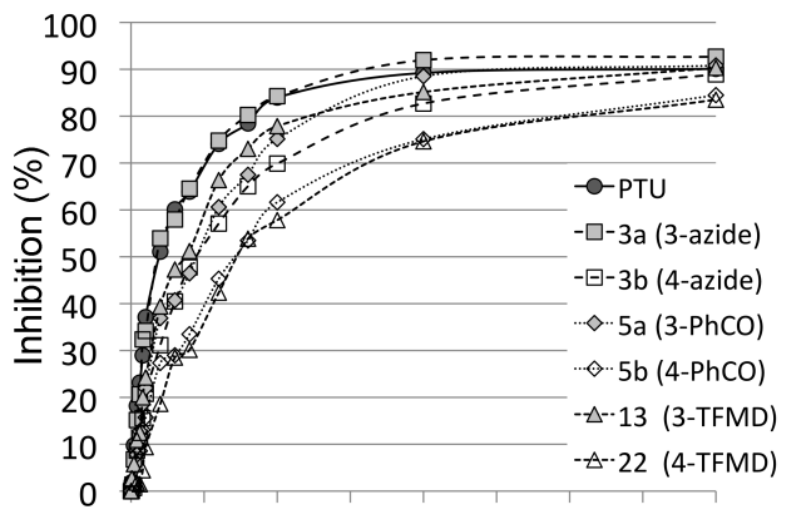

$\begin{array}{lllllllll}0 & 0.5 & 1 & 1.5 & 2 & 2.5 & 3 & 3.5 & 4\end{array}$

$\begin{array}{lllllllllll}0 & 0.1 & 0.2 & 0.3 & 0.4 & 0.5 & 0.6 & 0.7 & 0.8 & 0.9 & 1\end{array}$ [PTC derivative] $(\mu \mathrm{M})$

\section{B) DOPA}

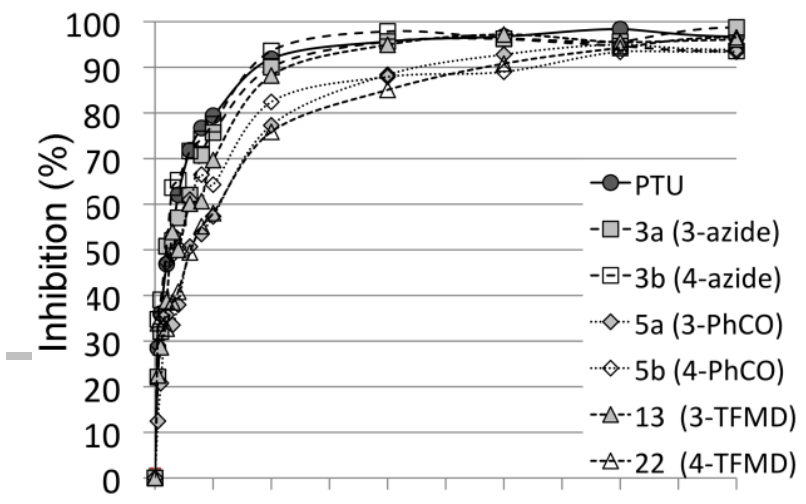

(Figure 2). Time-dependent decrease in maximum absorption at around $360 \mathrm{~nm}$ indicates that the synthetic compounds have enough photoreactivity for photoaffinity labeling. The azide derivatives ( $\mathbf{3} \mathbf{a}$ and $\mathbf{3 b})$ are also same reactivity, but benzophenone derivatives ( $5 \mathrm{a}$ and $\mathbf{5 c}$ ) are very low reactivity than these two photophores $(\mathrm{SI})$. These results are consistent with previous reports. ${ }^{[5]}$

Tyrosinase inhibitory activity assay: The synthesized photoreactive PTU derivatives were used as inhibitors in the tyrosinase assay for tyrosine and DOPA as substrates. The PTU derivatives that contained photophores at 3-position (3a, 5a, and 13), presented high inhibitions for tyrosine oxidation as same as mother skeleton PTU. Compounds with photophores introduced into the 4-position were less inhibitory (Figure 3, A). On the other hand, azide substitutions ( $\mathbf{3 a}$ and $\mathbf{3 b}$ ) resulted in inhibitory activity for DOPA oxidations that is higher than that achieved with the other two photophores (Figure 3, B). These results are consistent with substitution sites in the substrate were influenced for inhibitory activity of photoreavtive PTU derivatives. On the basis of these results, a detailed analysis of the the bioactivity of the photoreactive PTU derivatives was performed in addition to their functional analysis. $u$ [PTC derivative] $(\mu \mathrm{M})$ 
Figure 3. Inhibitiory activities of synthetic photoreactive PTU derivatives (3a, 3b, 5a, 5b, 13 and 22) for tyrosinase. ${ }^{[17]}$ Substrates: A) tyrosine and B) DOPA detailed conditions described in Experimental Section. Details of the inhibitory activities for all compounds, along with standard deviations, are summarized in the Supporting Information

\section{Conclusions}

The comprehensive synthesis of photoaffinity label reagents for PTU is firstly reported. Re-investigation for the synthesis of azide and benzophenone derivatives, establishments of the synthesis 3- and 4- aminophenyl-TFMD, and urea conversions from anilne derivatives based on the stabilities of the compounds are also reported. The photoreactive PTU derivatives have identical activities for mother compound PTU. These results indicate that the photoreactive PTU has enough affinity, allowing its use in the functional analysis of physiological conditions such as eating disorders. Such analysis ultimately provides an understanding of the underlying molecular mechanisms of biological processes.

\section{Supporting Information Summary Paragraph}

Experimental section, $1 \mathrm{H}$ and $13 \mathrm{C}$ NMR spectra, photolysis of azide- and benzophenone derivatives, and kinetic investigation of tyrosinase assay.

\section{Acknowledgements}

Part of this work was performed under the Cooperative Research Program of "Network Joint Research Center for Materials and Devices".

Keywords: Benzophenone $•$ Diazirine $•$ Phenylazide • Phenylthiourea $\bullet$ Photoaffinity label

[1] R. R. Mayrand, D. G. Levitt, J. Gen. Physiol., 1983, 81, 221-237.

[2] U. K. Kim, D. Drayna, Clin. Genet., 2004, 67, 275-280.

[3] a) F. Bernheim, M. L. C. Bernheim, J. Biol. Chem., 1942, 145, 213-217: b) A. D. Ryazanova, A. A. Alekseev, I. A. Slepneva, J. Enzyme Inhib. Med. Chem., 2012, 27, 78-83: c) P. Thanigaimalai, K.-C. Lee, V. K. Sharma, C. Joo, W.-J. Cho, E. Roh, Y. Kim, S.-H. Jung, Bioorg. Med. Chem. Lett., 2011, 21, 6824-6828.

[4] S. Ito, Pigment Cell Res., 2003, 16, 230-236.

[5] a) J. Brunner, Annu. Rev. Biochem., 1993, 62, 483-514; b) T. Tomohiro, M. Hashimoto, Y. Hatanaka, Chem. Records, 2005, 5, 385-395; c) M. Hashimoto, Y. Hatanaka, Eur. J. Org. Chem., 2008, 2513-2523; d) Y. Hatanaka, Chem. Pharm. Bull., 2015, 63, 1-12.

[6] H. Lamotte, F. Degeilh, B. Rousseau, P. Neau, P. Ripoche, Bioorg. Med. Chem. Lett., 1993, 3, 2587-2592.

[7] H. Lamotte, F. Degeilh, P. Neau, P. Ripoche, B. Rousseau, J. Labelled Compd. Radiopharm., 1994, 34, 289-295.

[8] E. M. Kamel, R. A. Ahmad, O. S. Moustafa, J. Chin. Chem. Soc., 2005 52, 149-153.

[9] M. P. Hay, S. Turcotte, J. U. Flanagan, M. Bonnet, D. A. Chan, P. D. Sutphin, P. Nguyen, A. J. Giaccia, W. A. Denny, J. Med. Chem., 2010 53, 787-797.

[10] J. Brunner, G. Semenza, Biochemistry, 1981, 20, 7174-7182.

[11] a) M. Dolder, H. Michel, H. Sigrist, J. Prot. Chem., 1990, 9, 407-415: b) H. Sigrist, M. Mühlemann, M. Dolder, J. Photochem. Photobiol. B, 1990 7, 277-287: c) T. Döring, P. Mitchell, M. Osswald, D. Bochkariov, R.
Brimacombe, EMBO J., 1994, 13, 2677-2685: d) A. Collioud, J. -F. Clémence, M. Sänger, H. Sigrist, Bioconjugate Chem., 1993, 4, 528536.

[12] B. Biasotti, S. Dallavalle, L. Merlini, C. Farina, S. Gagliardi, C. Parini, P. Belfiore, Bioorg. Med. Chem., 2003, 11, 2247-2254

[13] a) L. Wang, T. Yoshida, Y. Muto, Y. Murai, Z. P. Tachrim, A. Ishida, S. Nakagawa, Y. Sakihama, Y. Hashidoko, K. Masuda, Y. Hatanaka, M. Hashimoto, Eur. J. Org. Chem., 2015, 3129-3134: b) Y. Murai, K. Masuda, Y. Sakihama, Y. Hashidoko, Y. Hatanaka, M. Hashimoto, J. Org. Chem., 2012, 77, 8581-8587: c) Y. Hatanaka, M. Hashimoto, H. Kurihara, H. Nakayama, Y. Kanaoka, J. Org. Chem., 1994, 59, 383-387.

[14] L. Wang, Y. Murai, T. Yoshida, A. Ishida, K. Masuda, Y. Sakihama, Y. Hashidoko, Y. Hatanaka, M. Hashimoto, Org. Lett., 2015, 17, 616-619

[15] B. Zhu, H. Zhang, S. Pan, C. Wang, J. Ge, J. -S. Lee, S. Q. Yao, Chem. Eur. J., 2016, 22, 7824-7836

[16] M. M. Kremlev, A. I. Mushta, W. Tyrra, D. Naumann, H. T. M. Fischer, Y L. Yagupolskii, J. Fluorine Chem., 2007, 128,1385-1389.

[17] Details of the inhibitory activities for all compounds along with standard deviation, are summarized in the Supporting Information. 
Entry for the Table of Contents (Please choose one layout)

Layout 1:

\section{FULL PAPER}

Text for Table of Contents

Layout 2:

\section{FULL PAPER}

Author(s), Corresponding Author(s)*

Page No. - Page No.

Title

((Insert TOC Graphic here: max. width: $5.5 \mathrm{~cm}$; max. height: 5.0 $\mathrm{cm})$ )<smiles>[R][Y10]1cccc(NC(N)=S)c1</smiles>

Comprehensive synthesis of photoreactive phenylthiourea derivatives were achieved to elucidate photoaffinity labelling. The synthesized compounds were subjected to tyrosinase.

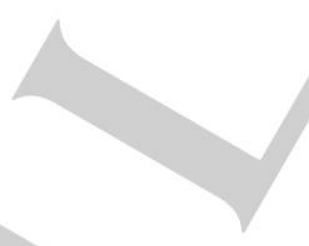

Akiko Ishida, Lei Wang, Zetryana Puteri Tachrim, Takeyuki Suzuki, Yasuko Sakihama, Yasuyuki Hashidoko, and Makoto Hashimoto*

Page No. - Page No.

Comprehensive Synthesis of Photoreactive Phenylthiourea Derivatives for the Photoaffinity Labeling

Keywords: Photoaffinity label / Phenylthiourea / Phenylazide / Benzophenone / Diazirine

(KeyTopics: Heterocycles) 